\title{
Derechos de las mujeres privadas de libertad. El caso Juana María de Lara en Ciudad del Este
}

\section{Rights of women deprived of freedom. The case of Juana María de Lara in Ciudad del Este}

\author{
Airym Monroy Sarta ${ }^{a}$, Claudia Avila Schmalko ${ }^{a}$
}

\begin{abstract}
Resumen
Este estudio registra las condiciones de vida de las mujeres privadas de libertad en las cárceles y las vulneraciones en razón de su género, condición laboral y privación de libertad. Describe la situación de los derechos sexuales y reproductivos de las mujeres privadas de libertad en la penitenciaría Juana María de Lara de Ciudad del Este 20162017 y fue extraído de la investigación Análisis de las condiciones de trabajo de las mujeres privadas de libertad en la cárcel Juana María de Lara de Ciudad del Este, Paraguay, presentada a la Universidad Nacional de Asunción como tesis para la obtención de título de grado en el año 2017. Hacen parte de los resultados la caracterización de la vida en la cárcel e información relacionada con el género en privación de libertad, en especial datos que evidencian el incumplimiento y falta de garantías que ofrecen las cárceles a las mujeres para ejercer libremente su sexualidad y decidir acerca de su reproducción. También presenta los factores de género identificados por las mujeres en relación con su trabajo, las condiciones para el mantenimiento de vínculos sociales y familiares, y el fuero de maternidad; la teoría de género estructura el análisis como una dimensión transversal del diseño de las conclusiones. Las mujeres están presentes como sujetos y objetos de la investigación en pro de generar estudios de género acerca de realidades paraguayas que tengan en cuenta las particularidades de las mujeres en el contexto de encierro, condiciones que devienen de la desigualdad estructural e histórica que afecta a las mujeres. Las conclusiones muestran algunas formas patriarcales que permiten al sistema de justicia configurar el control de la penitenciaría sobre el cuerpo de las mujeres privadas de libertad.
\end{abstract}

Palabras clave: género, privación de libertad, criminología feminista.

\footnotetext{
Abstract

This study records the living conditions of women deprived of liberty in prison and vulnerability on grounds of their gender, labor status
}

a Universidad Nacional de Asunción, Facultad de Ciencias Sociales, Paraguay.

Correspondencia a: anissarta17@gmail.com

Recibido:

13 ocubre 2018

Aceptado:

6 diciembre 2018

Artículo publicado en acceso abierto bajo la Licencia Creative Commons.

c) (i)

Cita:

Monroy Sarta, A., \& Avila

Schmalko, C. (2018).

Derechos de las mujeres privadas de libertad. El caso Juana María de Lara en Ciudad del Este. Kera Yvoty: reflexiones sobre la cuestion social, 3, 54-63. 
and deprivation of liberty. Describe the situation of sexual and reproductive outcomes of women deprived of liberty in prison Juana María de Lara de Ciudad del Este 20162017 and was extracted from research Investigation of conditions of work of deprived girls in prison Juana María de Lara from Ciudad del Este, Paraguay, presented to the National University of Asunción as a thesis to obtain a degree degree in 2017. There are also part of the results of the characterization of life in the carcass and related information in deprivation of liberty, in particular data showing evidence of default and the lack of guarantees that it will provide prisoners with women to freely choose their sexuality and decide on their reproduction. También presents the gender factors identified by women in relation to their work, the conditions for maintaining social and family ties, and the motherhood child; the theory of gender structure and analysis as a cross-sectional dimension of the conclusions drawn. Women are present as objects and objects of research in order to generate gender studies about Paraguayan realities that have in mind the particularities of women in the context of the context, conditions that have to do with the structural and historical inequality that affects them. women. The conclusions are also some patriarchal forms that allow the justice system to configure penitentiary control over the body of women deprived of liberation.

Keywords: gender, liberation deprivation, feminist criminology.

\section{Introducción}

En 2016 las mujeres privadas de libertad eran 821, lo que representa el $6 \%$ de la población penitenciaria. Esta diferencia en números hace que las mujeres privadas de su libertad se encuentren en desventaja al momento de ser vistas por el Estado como grupo que necesita de políticas públicas y cumplimiento de sus derechos (Von Lepel, 2016).

La situación de hacinamiento y sobrepoblación de las cárceles paraguayas se suma a la incapacidad de las instituciones de proveer no solo de un espacio físico apto para la privación de libertad, sino también insumos básicos para la reproducción de la vida como medicamentos, elementos de higiene, materiales de ocio e incluso comida.

Durante el año 2015, en los dos penales de mujeres más poblados en el país, Buen Pastor y Juana María de Lara, se detectaron vulneraciones diferenciales a los derechos de las mujeres. "No accedían a atención médica especializada o diferencial, ni de salud mental, no había servicios de ginecología ni mastología, no se las proveía de toallas higiénicas, y tenían dificultades de acceso al agua. Además, el régimen de visitas era mucho más estricto que el de los hombres" (Leguizamón, 2015, p. 305).

Esta es la realidad de las cárceles de mujeres: dentro del sistema penal las mujeres deben afrontar el estigma de romper con el rol asignado por la sociedad como esposas, madres, cuidadoras, sumado a la responsabilidad de hacerse cargo, a causa de la falta de políticas públicas, de realidades como la de ser madres lactantes o la responsabilidad del cuidado de los hijos/as durante el encarcelamiento.

$$
\text { Las prisiones asignadas }
$$

exclusivamente a mujeres están ubicadas generalmente lejos de sus hogares, debido a las dimensiones de la población respecto a los hombres. En definitiva, una de las principales necesidades de las mujeres - la del mantenimiento de los vínculos familiares - se ve gravemente comprometida.

Dentro del contexto de privación de libertad, el acceso a un trabajo asalariado se presenta como una alternativa válida e indispensable, pues implica recibir dinero trabajando durante el tiempo de reclusión. Por esta razón la experiencia de las trabajadoras de Fujikura es un caso paradigmático, presentado con mucha cobertura mediática como una solución de reinserción laboral para la que el Estado generó en tiempo récord un Plan de Atención a Mujeres Privadas de Libertad. Dicho plan solo sirvió para aplicar reformas en la 
penitenciaría, que firmó un convenio con la empresa presentado como "un ejemplo de responsabilidad social del sector privado" ${ }^{1}$.

La investigación que da origen a este informe ${ }^{2}$ es un estudio exploratorio, motivada principalmente por la curiosidad de conocer las condiciones de trabajo que ofrecía la "primera cárcel industrial de mujeres del país”, habilitada en la penitenciaría de mujeres de Ciudad del Este, Juana María de Lara, en el año $2015^{3}$. Ese trabajo tuvo como fuente de referencia para la identificación de las variables las perspectivas de las mujeres, registradas en las entrevistas.

Para el presente informe se han excluido algunos de los datos referentes al proceso de trabajo de las mujeres, su relación con la empresa y las condiciones de trabajo con el objetivo de profundizar los aspectos relacionados con la sexualidad y la reproducción.

El estudio plantea ver a las mujeres participantes de la investigación como sujeto y objeto de estudio desde las particularidades del caso reconociendo las condiciones estructurales de desigualdad entre hombres y mujeres en la sociedad que han configurado la realidad que hoy viven las mujeres en Paraguay, condiciones que se recrudecen al entrar en el sistema penal. "El feminismo, en su dimensión intelectual,

1 El Ministerio de Justicia en el 1zer. Congreso de las Naciones Unidas contra la Droga y el Delito, realizado en la ciudad de Doha, Qatar, presentó al emprendimiento como un ejemplo en políticas de reinserción: http://www.ministeriodejusticia.gov. py/index.php/noticias/presidente-de-la-republicahabilito-el-primer-penal-industrial-de-mujeres-delparaguay

2 El presente informe de investigación es un extracto de los resultados de una tesis de grado presentada para obtener el título de licenciatura en Trabajo Social en el año 2017 por Claudia Avila Schmalko y Airym Sarta.

3 Ver en: http://www.ultimahora.com/inauguranprimera-carcel-industrial-mujeres-ciudad-deleste-n905473.html; http://www.lanacion.com. py/2015/o6/15/este-martes-se-inaugura-la-primeracarcel-industrial-de-mujeres-del-paraguay/; http:// www.presidencia.gov.py/noticia/17348-jefe-deestado-habilito-primera-carcel-industrial-demujeres-en-ciudad-del-este.html\#.WEmCqtXhDIU ha mostrado que el conocimiento está situado históricamente y que cuando un colectivo social está ausente como sujeto y como objeto de la investigación, a ese conocimiento le falta objetividad científica y le sobra mistificación" (Cobo, 2005, p. 251).

Teniendo en cuenta la realidad a estudiar y considerando el marco teórico que sustenta las dimensiones establecidas se plantea responder a las preguntas: ¿Cómo son las condiciones de vida en la penitenciaría Juana María de Lara? ¿Existen factores vinculados al género que afectan a las mujeres en privación de libertad?

\section{Objetivos \\ General}

Analizar las condiciones de vida de las mujeres privadas de libertad que prestan servicios a la empresa Fujikura en la Penitenciaría Juana María de Lara, en Ciudad del Este, entre 2016 y 2017.

\section{Específicos}

1. Describir las condiciones de vida de las mujeres privadas de libertad.

2. Identificar factores vinculados al género que afectan directamente las condiciones de vida de las mujeres en la Penitenciaría Juana María de Lara.

\section{Materiales y métodos}

La investigación es un estudio cualitativo, de corte transversal utilizando el estudio de casos. Para el diseño metodológico se cuenta con entrevistas a informantes clave durante los años 2015 y 2016. La población de estudio son las 20 mujeres privadas de libertad en la Penitenciaría Juana María de Lara que entre el año 2016 y 2017 trabajan para la empresa Fujikura Automotive S. A.

La muestra fue homogénea: los datos se recogieron de un grupo de características similares (condición laboral, privación de libertad). El tamaño de la muestra fue del $40 \%$ del universo total. Se hicieron cuatro entrevistas a grupos de dos mujeres cada 
vez. Para la selección de la muestra se tuvo en cuenta, además de la condición de trabajo, a mujeres con diferentes meses de antigüedad en el trabajo y a mujeres de diferentes edades y ciudades de procedencia.

Para las entrevistas se utilizó una guía semiestructurada que fue ordenada con la idea de avanzar poco a poco en los temas y ganar confianza para hablar abiertamente con las entrevistadas pese a las condiciones que implica una entrevista en contexto de encierro.

\section{Consideraciones éticas}

Las entrevistas fueron realizadas dentro de un marco de privacidad con las internas previo contacto con la directora para evitar represalias. La información personal: nombres, apellidos, edad, situación procesal no fueron incluidos en la entrevista ni registrados, para preservar su anonimato.

Las mujeres entrevistadas fueron informadas de la intención de la investigación y el anonimato durante la recolección de datos y la redacción de los resultados. También fueron consultadas para decidir ser parte de la investigación y si se podía grabar o no sus respuestas. Optamos por establecer un consentimiento verbal de parte de las entrevistadas y no escrito para mantener un ambiente de distención y generar una relación de mayor horizontalidad dada la situación especial que implica el contexto de encierro.

\section{Resultados}

En diciembre del 2016 según el Censo Penitenciario del Mecanismo Nacional de Prevención contra la Tortura y Otros Tratos o Penas Crueles, Inhumanos o Degradantes (MNP) se registraban 107 mujeres privadas de libertad en la Juana María de Lara, recluidas sin distinción entre procesadas (82) y condenadas (25). La capacidad de albergue era de 66 personas, sobrepasado por 41 mujeres en el momento de la toma de datos. El edificio de la penitenciaría tiene dos oficinas, un pabellón central de
11 celdas, un patio pequeño y una celda de aislamiento. Al lado derecho del pabellón de celdas hay una cantina, una intendencia y un tinglado grande. Frente al tinglado hay un patio con cancha de voleibol. Detrás, las salas usadas para visitas y salas de talleres; la más amplia de estas la usa Fujikura.

Cada baño es utilizado por dieciséis a diecisiete mujeres, los baños no cuentan con agua caliente porque la instalación eléctrica no está permitida por la administración para impedir la carga de celulares, que están prohibidos. En los baños sin duchas, las mujeres usan baldes para bañarse, los sanitarios y sus instalaciones están deteriorados.

El 92,9 \% de las mujeres dijo tener elementos de higiene y aseo personal. Quienes los tienen solamente el 5,6\% los reciben de la penitenciaria. En el 94\% de los casos son las mujeres mismas o sus familias quienes los adquieren.

Las celdas tienen literas de madera, aquellas que no tienen cama duermen en colchones en el piso. Cada celda cuenta con un mueble de madera para guardar sus pertenencias, pero no dan abasto, por lo que muchas de las mujeres guardan sus pertenencias en bolsas. Cada celda tiene un televisor de uso común.

Existe un pabellón destinado, según la Dirección, a la tercera edad. Diez mujeres en dos celdas comparten ese espacio. Cada cierto tiempo y de forma arbitraria se las cambia de celdas separando incluso a madres e hijas.

Más del $80 \%$ dijo tener cucarachas, mosquitos, hormigas en su celda y el $8,2 \%$ roedores, ratas, o comadrejas. La limpieza de las celdas está a cargo de las internas. El $87,4 \%$ cuenta con elementos para limpiar su celda, el $71,6 \%$ debe comprarlos, $23 \%$ de ellas los tiene porque sus familiares los proveen y solo un $5,4 \%$ son provistos por la institución.

Las funcionarias en contacto con las mujeres privadas de libertad son, además de la oficial de guardia y la directora, tres agentes penitenciarias en turnos para las 24 horas del día, además de una abogada. No 
había en el momento de la toma de datos especialistas de la salud trabajando en la penitenciaría.

Características comunes del grupo de mujeres trabajadoras: son madres solteras, no reciben visitas, ya sean familiares o privadas, y tienen entre 20 y 35 años. Con respecto a la edad, las mujeres creen que podría ser excluyente para mujeres mayores porque todo el trabajo se hace de pie durante las 7,5 horas de la jornada. En relación con las visitas, asumen que el hecho de trabajar en Fujikura limita los días de visita porque la empresa no se arriesgaría a contratar gente que recibe visitas (íntimas o familiares), ya que eso sube las probabilidades de que las mismas se ausenten del trabajo los días de visita entre semana (martes y jueves).

\section{Visitas}

El acceso a las visitas familiares, en lugar de ser garantizado o resguardado por la penitenciaría, es de alguna forma desalentado por los controles que se realizan para acceder a ellas, estos son extralimitados e incluso incumplen normas internacionales relacionadas con estos procesos. Según los datos del censo el $92.3 \%$ de las personas que visitan a las mujeres se quejan de las revisiones que se realizan para ingresar a la penitenciaria, uno de los porcentajes más altos a nivel nacional. Esto podría estar causando que las familias no visiten a las internas, quitándoles a una gran mayoría la posibilidad de acceder a bienes de consumo que requieren en el día a día.

Y si no trabajamos también nosotras que no tenemos visita, yo y ella no tenemos visitas por ejemplo y entonces tenemos que trabajar es la única opción porque tenemos nuestros gastos personales y todo eso.

Los controles generan molestias en los familiares, en primer lugar, porque es obligatorio desnudarse para acceder a la penitenciaría, a los niños y niñas se les quita la ropa también, incluido el pañal.
El registro en todo el cuerpo, según los visitantes es abusivo en las partes íntimas. Se denunciaron hechos como la no utilización de guantes en las revisiones íntimas, la obligación de hacer sentadillas como parte del control incluso para embarazadas y que a las mujeres en periodo menstrual no se les permita ingresar usando toalla higiénica.

A las visitas se les prohíbe ingresar ropa nueva, alimentos envasados en cartón, café o caramelos. Una de las conjeturas respecto a esa medida es que los productos se venden en la cantina, y en el caso de la ropa nueva estaría relacionado con que las funcionarias venden estos productos a las internas. Las ropas o bebidas no figuran dentro de las prohibiciones establecidas en el código de ejecución penal.

En el momento de la visita, las mujeres y sus familiares (incluyendo parejas) tienen restricciones de demostración de afecto como sentarse muy cerca, tomarse de las manos, abrazarse y sobre todo besarse. Para el momento de recibir a la visita está prohibido también el uso de shorts; blusas abiertas, con escotes, o faldas cortas.

Para que las personas puedan disponer de mesa y sillas para recibir a sus visitas deben alquilarlas, además los baños para el uso de las visitas están en muy mal estado.

En cuanto al acceso a visitas íntimas, los requisitos son mucho más restrictivos que en otros penales. Las mujeres deben estar casadas, presentar certificados de matrimonio o concubinato y los resultados de análisis de laboratorio de la interna y su pareja (únicamente) del sexo opuesto. Para dar una idea de la política de la institución, una de las piezas, que se suponía era para visitas privadas, fue destinada a mujeres con tuberculosis.

\footnotetext{
4 El Comité contra la tortura en relación con las prácticas de tortura o tratos crueles en su informe del 2017 mostró preocupación por la aplicación de registros intrusivos vaginales y actos de desnudez forzada como medio de control de seguridad en centros penitenciarios de mujeres, si bien aprecia las informaciones proporcionadas por la delegación respecto a los esfuerzos para sustituir estas medidas por detectores de metales (arts. 2, 12 y 16).
} 
El monto para acceder por dos horas a la habitación privada es de 20 ooo guaraníes, que se abona a la jefa de guardias. En otras penitenciarias las mujeres pueden acceder a las visitas íntimas con sus parejas sentimentales estables luego de un plazo, la regla establecida en Ciudad del Este implica que gran parte de ellas no puedan acceder al derecho de visitas, por lo cual reclaman constantemente que pierden a sus parejas por no poder recibirlas.

\section{Salud sexual y reproductiva}

El acceso a salud sexual en la penitenciaria, según lo relevado por el censo, era realmente preocupante: el $84,8 \%$ de las mujeres respondió que no se realizan controles ginecológicos dentro de la cárcel y de las que han accedido algunas no conocen sus resultados.

Un tema recurrente en las entrevistas es la limitación en el acceso a visitas privadas, relacionado por las internas con el acceso al trabajo estable y con el hecho de ser mujeres.

Pero es luego demasiado trámite para entrar acá y no da gusto. No es como los hombres acá.

Las visitas privadas son parte de los derechos sexuales y reproductivos de las mujeres, y es un derecho al que no acceden, pues se les exige la presentación de certificados de matrimonio, trámites médicos, y un pago decidido por la Dirección. Para la mayoría de las mujeres, intentar acceder a una visita privada es frustrante porque, si recibir a su pareja legal en un recinto destinado para eso depende de tener recursos y un acompañamiento legal, es excluyente para la mayoría de ellas.

todos los días hay privada si tenés pareja, si vos tenés tu pareja, vas a hacer, si tenés abogado y todas esas cosas, pero si es así de pedir nomás, no.

Muchas de las entrevistadas se refirieron a la privada como si no existiera, ya que las posibilidades de acceder a este derecho son para ellas completamente nulas.

No, nosotros no tenemos privada. Tenés que ser casada, yo hace 10 meses por ahí que no.

En los relatos está presente la necesidad de acceder a estas visitas, ninguna lo presentó como un tabú, aunque algunas manifestaron haber renunciado a la posibilidad de las visitas privadas y sentirse disgustadas por la situación porque, ante tantos obstáculos, han decidido no intentarlo.

Los testimonios reconocen esta situación como una particularidad de las cárceles de mujeres, una clara diferencia para las entrevistadas en relación con los derechos que tienen los hombres privados de su libertad. Esta diferencia se agudiza en esta penitenciaría en particular, la cual tiene controles extremadamente estrictos sobre los cuerpos de estas mujeres: quienes logran superar los límites para ingresar a la visita son condicionadas por la Dirección de la penitenciaria a aplicarse anticonceptivos con regularidad cuando acceden a las visitas privadas. Contradictoriamente, el acceso a los anticonceptivos en la penitenciaría de manera voluntaria por medio de la sanidad no es posible y en caso de serlo tiene altos costos.

El control en torno a las privadas aparece ligado al ingreso a la empresa, o la posibilidad de trabajar ahí. Las mujeres lo mencionaron como una característica de las trabajadoras.

En relación con el acceso de las mujeres a toallitas higiénicas o tampones, según el censo el 73,2\% de las mujeres cuenta con estos elementos, pero en su mayoría son comprados por las mujeres dentro del penal, proveídas por sus familiares, y en menor parte por el penal.

\section{Fuero de maternidad}

Para establecer las garantías de las mujeres trabajadoras, en la investigación se tuvo en cuenta el fuero de maternidad, un elemento indiscutible en torno al género 
en el mundo laboral. Cuando preguntamos a las mujeres sobre la posibilidad de tener reposo de maternidad, la primera respuesta por parte de la mayoría de ellas fue que no existe posibilidad de embarazarse en la penitenciaría.

iiNo!! Acá nadie se embaraza. No, en serio, nosotros no tenemos privada.

No, acá no. Allá afuera sí. Acá directamente salís o le das espacio a otra.

Al ver que esta no era una posibilidad en absoluto para ellas y que desde la inauguración en 2015 no había ningún caso de embarazo de las trabajadoras dentro de la penitenciaria, consultamos si en algún momento les hablaron de esto al contratarlas, o estaba en su contrato. La respuesta fue:

No, pero no tenemos luego novio.

La posibilidad de acceder a un reposo de maternidad estaba, para una de las internas, relacionada a la orientación sexual de sus compañeras:

Acá lo que hay mas mucho es tortilleras, lesbiana demasiado mucho hay. Repleto está, y la lesbiana no se puede embarazar, es la realidad.

Las mujeres privadas de libertad entrevistadas, además de no tener fuero de maternidad, en las condiciones de reclusión a las que están sometidas, ni siquiera pueden decidir si embarazarse o no. En la tesis se incluyeron testimonios referentes a dos casos en los que los anticonceptivos les fueron aplicados sin que ellas lo pidieran ${ }^{5}$.

5 De comprobarse esto, se estaría incumpliendo directamente el artículo 61 de la constitución de la República: "De la planificación familiar y de la salud materno-infantil" dondeel Estadoreconoceel derecho
Según el Censo Nacional, las penitenciarías con mayor cantidad de mujeres embarazadas son la de San Pedro (37,5\% del total), Encarnación (25\%) y Coronel Oviedo (15,4\%). La penitenciaria Juana María de Lara era la única que reportaba no tener mujeres embarazadas en 2016, según su directora porque "no están dadas las condiciones" ${ }^{\circ}$.

\section{Vínculos familiares y sociales}

En relación con los vínculos familiares y sociales que las mujeres privadas de libertad tenían antes de entrar al sistema penitenciario, de la cárcel Juana María de Lara el 18,4\% de las internas tenía algún adulto mayor a su cargo antes del ingreso. A nivel nacional el 6,6\% de las mujeres privadas de libertad se encuentra recluida con su hijo/ay, de estas madres, el 96,8\% vive con un hijo/a y el 3,2\% con 2 hijos/as.

Durante su detención, la cercanía de familiares que alguna vez tuvieron a cargo las mujeres es un criterio crucial para que después de la condena puedan reinsertarse a redes de contención y no reincidan, por eso que los vínculos son incluidos como un factor de género. En el grupo de las trabajadoras de Fujikura, se analizó de qué manera se garantizó la cercanía a las personas con las que tuvieron un vínculo anterior, que cumple un papel importante en la salud mental de las personas en contexto de encierro.

En la Juana María de Lara, ninguna de las internas vive con ningún niño/a dentro del penal a pesardeserestauna penitenciaría exclusiva de mujeres y de que el $85,2 \%$ del total de las internas de la penitenciaría

de las personas a decidir libre y responsablemente el número y la frecuencia del nacimiento de sus hijos, así como a recibir, en coordinación con los organismos pertinentes, educación, orientación científica y servicios adecuados en la materia.

6 Las Reglas de Bangkok, adoptadas por el Estado paraguayo mediante la Acordada N.o 845/2013 de la Corte Suprema de Justicia, incluyen consideraciones que garantizan el trato equitativo y justo durante el proceso de detención, sentencia y encarcelamiento prestándose especial atención a las situaciones derivadas del embarazo y el cuidado de los niños/as. 
es madre. El 95\% de las trabajadoras de Fujikura tienen al menos un hijo, lo cual es un dato resaltante de desvinculación: ninguna de ellas vive con sus hijos menores de diez años en el penal, aun cuando solían estar a su cuidado. Según Atabay (2013) es fundamental tener en cuenta el interés del niño/a y dar preferencia a otras alternativas de detención y encarcelamiento en los casos de las mujeres embarazadas y mujeres con niños/as pequeños/as a su cargo.

Lo que sucede en la Juana María de Lara es que, a pesar de ser una cárcel exclusiva para mujeres, no cuenta con espacios adecuados para que las mujeres puedan estar con hijos/as pequeños/as. En el censo se registró que las visitas son un $89.5 \%$ de sus familiares; el $34 \%$ son semanales; el 18\%, cada 15 o 22 días; y el 13\% visitas mensuales. El 10,7\% de las mujeres dice no recibir visitas, $50 \%$ declaran que es por la distancia y el $25 \%$ por motivos económicos de su familia. Las madres son derivadas al penal Buen Pastor de Asunción con los hijos o van a entidades de abrigo de Alto Paraná, en caso de no haber familiares que puedan hacerse cargo de los niños/as.

Ante las desagradables situaciones que pasan los familiares para acceder a visitar a las mujeres privadas de libertad en la cárcel estudiada, y pese a estos obstáculos las familias acuden con relativa frecuencia a la visita. Creemos que se debe a que, para quienes no trabajan dentro de la cárcel, recibir dinero en la visita familiar es la única manera de poder satisfacer las necesidades no cubiertas por la administración penitenciaria: ropa, comida, artículos de higiene personal y de limpieza. En la cárcel es necesario tener dinero incluso para tomar mate o comprar hielo, debido al excesivo control que tienen sobre las pertenencias de las internas y el manejo de la electricidad.

En relación con la comunicación telefónica en la Juana María de Lara encuentra muchas dificultades: se reiteraba en los informes de seguimiento del MNP del año 2015 y 2016 el pedido de adoptar medidas que faciliten el contacto de las internas con sus familiares y defensores, pues el permiso para el uso del teléfono depende de la voluntad de la directora o de la jefa de guardia, al no existir un procedimiento formal al respecto. Según las reglas (comunicadas verbalmente a las internas) las comunicaciones no pueden durar más de un minuto, solo los días miércoles, un intento por número y cuando está la Directora presente.

\section{El trabajo}

Las

mujeres entrevistadas identificaron como requisito para acceder al trabajo saber leer, escribir, no estar medicada, haber pasado por la selección de la empresa (exámenes de memoria y agilidad motriz) y tener la "venia de la directora". Al preguntarle acerca de este requisito a la directora, señaló que es necesario no tener llamados de atención y presentar buena conducta según criterios establecidos por ella en el reglamento.

Las trabajadoras de Fujikura tienen una jornada de trabajo de 7:00 a 15:30 horas, de lunes a sábado. Reciben por este trabajo semanalmente 178.500 guaraníes, equivalente a 35.700 por jornal. Pueden ausentarse para asistir a controles médicos o audiencias judiciales con previo aviso y sin remuneración por los días no trabajados. No cuentan con reposo médico pago.

En las entrevistas se destaca como respuesta la relación entre el acceso al trabajo y la capacidad necesaria para su ejecución con el hecho de ser "madres solteras". Las respuestas relacionadas con la maternidad, más allá de ser copia del discurso de la empresa en torno a la contratación de mujeres en sus plantas, muestran que ellas mismas se proyectan con necesidades diferentes a quienes no son madres. Incluso en relación con la contratación de hombres, las mujeres vinculaban el interés de la empresa por "ayudar" a las mujeres madres y jóvenes como principal razón para contratarlas a ellas, por encima de las capacidades o el entrenamiento.

Yo, por ejemplo, si yo salgo de acá, si me dan ese trabajo, le agradecería 
muchísimo porque hay muchas madres solteras que quieren trabajar, y no tiene la oportunidad. Y, en cambio, ahí vos no necesitás que estudies ni nada, solamente te enseñan cómo lo que debés hacer.

Yo creo que ocupan mujeres porque las mujeres son más necesitadas y porque hay muchas madres solteras que necesitan y necesitan ayudar a su familia y sus hijos, más queremos trabajo, los hombres no.

\section{Conclusiones}

Las necesidades específicas de las mujeres privadas de libertad, contempladas en el marco legal, son ignoradas por las direcciones de las cárceles y poco atendidas por el sistema judicial.

De esta manera la falta de garantías en el acceso a derechos básicos expone a las mujeres a pasar toda su vida en situación de desventaja sin poder insertarse a una vida posterior a la condena. La privación de libertad dificulta que las mujeres accedan a derechos aún más cuando concurre alguna otra causa de vulnerabilidad: edad, pobreza, discapacidad, orientación sexual, migración, entre otras ${ }^{7}$.

Cuando de parte del Estado, quien es el responsable de estas mujeres en la reclusión, no reciben las garantías mínimas para la reproducción de su vida: comida, elementos de limpieza personal e incluso elementos de limpieza de las celdas - que son de propiedad estatal - las mujeres se ven prácticamente obligadas a trabajar para poder sobrevivir durante la privación de su libertad.

Las entrevistadas se encontraban trabajando antes de ingresar a la penitenciaria en diversos oficios o habían trabajado dentro de la penitenciaria. Las condiciones de vida en la penitenciaría configuran que ese trabajo en condiciones

7 Instrumentos de derechos humanos como Las Cien Reglas de Brasilia, ratificadas según Acordada № 633/10 de la Corte Suprema de Justicia describen las garantías que deben darse en el acceso a justicia e incorporan precisamente como causas de vulnerabilidad el género y la privación de libertad (Reglas 8 y 10). de sobreexplotación (por la remuneración y modo de contrato) sea su única opción, que podría verse como la mejor, sólo dadas las condiciones que la cárcel les ofrece.

Toda la precariedad laboral que describimos se encuentra justificada en el imaginario de las mujeres por la posibilidad de ser contratadas en la empresa al obtener su libertad. Esta esperanza está reforzada por un discurso vinculado al género que tiene que ver con los mandatos sociales relacionados a la maternidad, pues es casi un requisito ser madre soltera para trabajar para Fujikura. Este discurso de protección de la maternidad es explícito por parte de la empresa, sus representantes abiertamente alegan que contratar madres solteras hace posible para ellos una baja rotación del personal, ya que las madres solteras tienen menos posibilidades de conseguir trabajo y se quedan trabajando para la planta industrial mucho tiempo ${ }^{8}$.

Las entrevistadas afirman que, al no recibir visitas, necesitan trabajar para poder mantener sus condiciones devida básicas, ya que el Estado no las garantiza. Esto presenta una paradoja, pues, aunque la empresa no les exija formalmente abandonar sus vínculos, negarse a la educación, la salud y las audiencias judiciales, si alguna vez acceden a alguna de estas, están en falta y se les descuenta el salario.

De la misma forma las faltas disciplinarias de la empresa están ligadas a las de la cárcel: la buena conducta es un concepto que depende de la subjetividad de la persona a cargo de la Dirección, que define las reglas de convivencia, visita y acceso a servicios de acuerdo a sus parámetros pese a las observaciones de las autoridades respecto a la arbitrariedad de estos controles.

El control que ejerce la penitenciaría sobre las mujeres es a través de disciplinamiento y el aislamiento. En la cárcel, la rutina sumada al control de las guardias y las prohibiciones en torno a

8 Ver en: http://www.abc.com.py/edicion-impresa/ suplementos/empresas-y-negocios/ibarrabuscamos-a-las-madres-solteras-589956.html 
las visitas familiares e íntimas aíslan a las mujeres aún más que la privación de libertad que ordena la condena judicial.

Dado el poco acceso a salud sexual y los límites a las decisiones sobre su reproducción (embarazarse o no) el control de la cárcel sobre el cuerpo de las mujeres excede lo previsto en el Código Penal, tendría que observarse si existe un control similar en las cárceles de hombres.

En el presente informe se incluye la información relacionada a derechos sexuales y reproductivos de las mujeres, que se ha preservado inédita como lo proponían las consideraciones éticas de la investigación de origen, para preservar el anonimato de las entrevistadas un tiempo que a las autoras ha parecido prudente. La decisión de publicarlo se debe a que la realidad de las cárceles de mujeres no ha cambiado en estos años y es imperiosa la necesidad de hablar públicamente del control que ejerce el poder punitivo sobre el cuerpo de las mujeres, recrudecido en la privación de libertad. Este es un tema que hoy en día, cuando se impone la censura a los estudios de género, se debe hablar alto y fuerte.

\section{Referencias Bibliográficas}

Atabay, T. (2013). Mujeres privadas de libertad: una guía para el monitoreo con perspectiva de género, 1-26. http:// goo.gl/hJ1HDc
Cobo, R. (2005). El género en las ciencias sociales. Cuadernos de Trabajo Social, 18, 249-258. https://doi.org/10.5209/ CUTS.8441

Leguizamón, D. (2015). Alarmante crecimiento de la población penitenciaria y pocas acciones para revertir este fenómeno. En CODEHUPY. (Ed.), Yvypóra Derécho Paraguáipe - Derechos Humanos en Paraguay 2015 (p. 299-315). Asunción: CODEHUPY.

MNP, Mecanismo Nacional de Prevención de la tortura y otros tratos o Penas Crueles. (2015). Informe anual de gestión. Asunción: Goiriz Imagen.

MNP, Mecanismo Nacional de Prevención de la tortura y otros tratos o Penas Crueles. (2016). Censo de mujeres privadas de libertad 2015. http://www. mnp.gov.py/index.php/investigacionsocial/2015-o 8-23-o 4-o 9-46/ Publicaciones/Censo-de-MujeresPrivadas-de-Libertad-2015/

Von Lepel, S. (2016). Las condiciones de privación de libertad en los centros penitenciarios constituyen un trato inhumano, cruel y degradante. En CODEHUPY. (Ed.), Derechos Humanos Paraguay (pp. 237-243). Asunción: Coordinadora de Derechos Humanos del Paraguay (CODEHUPY).

\section{Sobre los Autores}

Airym Monroy Sarta

Egresada de la carrera de Trabajo Social en el año 2017. Trabaja en educación y derechos humanos. Es intérprete de lengua de señas desde el año 2011. Ha colaborado en investigaciones relacionadas al impacto del modelo agroexportador en Paraguay y en sistematizaciones relacionadas a los derechos humanos de niños, niñas y adolescentes. Es activista por los derechos de las personas LGBTI.

\section{Claudia Avila Schmalko}

Graduada de la carrera de Trabajo Social en 2017. Ha coordinado investigaciones y proyectos dirigidos a formular políticas de ordenamiento territorial en Paraguay. Tiene experiencia en georeferenciación de información y mapeamiento con programas especializados. Cuenta con varias publicaciones, entre ellas el Atlas del Agronegocio en Paraguay, editado por BASE Investigaciones Sociales. 